\title{
Semiactive backstepping control for vibration attenuation in structures equipped with magnetorheological actuators
}

\author{
Ningsu Luo, Rodolfo Villamizar, Josep Vehí and Shirley Dyke
}

\begin{abstract}
This paper deals with the problem of semiactive vibration control of civil engineering structures subject to unknown external disturbances (for example, earthquakes, winds, etc.). Two kinds of semiactive controllers are proposed based on the backstepping control technique. The experimental setup used is a 6-story test structure equipped with shear-mode semiactive magnetorheological dampers being installed in the Washington University Structural Control and Earthquake Engineering Laboratory (WUSCEEL). The experimental results obtained have verified the effectiveness of the proposed control algorithms.
\end{abstract}

keyword: Smart structures, semiactive control, vibration attenuation, backstepping control, hysteretic damper.

\section{INTRODUCTION}

Significant advances in the vibration control of civil engineering structures have been achieved in the last years with the increasing application of emergent technologies and smart materials. Among diverse structural control techniques proposed (active, passive, semiactive, etc.), the semiactive control concept becomes very promising for the vibration attenuation in flexible structures due to its inherent stability, requirement of low electric supply and the facility of maintenance. In a semiactive control system, on-line adjustment of the damping and/or stiffness of adaptable devices are done according to feedback signals and control commands. In general, a semiactive controller can act in a desirable fashion in both passive and active control modes, with its performance generally enhanced in active mode [1][2]. One of promising semiactive actuators is the magnetorheological (MR) damper which can change rapidly its state from linear viscous fluid to semi-solid in milliseconds when exposed to a magnetic field. However, the design of semiactive controller are generally difficult due to the high nonlinearities, mainly the hysteresis phenomenon, presented

This work has been partially funded by the European Union (European Regional Development Fund) and the Commission of Science and Technology of Spain (CICYT) through the coordinated research projects DPI200508668-C03-02 and DPI2002-04018-C02-02 and by the government of Catalonia through SGR00296.

Ningsu Luo and Josep Vehí are with the Department of Electronics, Computer Science and Automation, Universitat de Girona, 17071, Girona, Spain. ningsu@eia.udg.es, vehi@eia.udg.es

Rodolfo Villamizar is with the School of Electrical and Electronics Engineering and Telecommunication, Universidad Industrial de Santander, Bucaramanga, Colombia. rovillameuis.edu.co

Shirley J. Dyke is with the Faculty of Engineering Sciences, Washington University in St.Louis, , USA. sdyke@seas . wust 1 . edu by the MR damper. In this paper, two new semiactive control approaches are proposed based on the backstepping control technique for attenuating vibrations of civil engineering structures subject to unknown external disturbances. The paper is organized as follows. First, the description of experimental setup is given. Then, the identification of hysteretic model of the MR damper is presented. In the controller design, the dynamics of MR dampers is taken into account so as to achieve the better performance of the semiactivelly controlled structure. Finally, experimental results are presented to show the effectiveness of proposed control schemes.

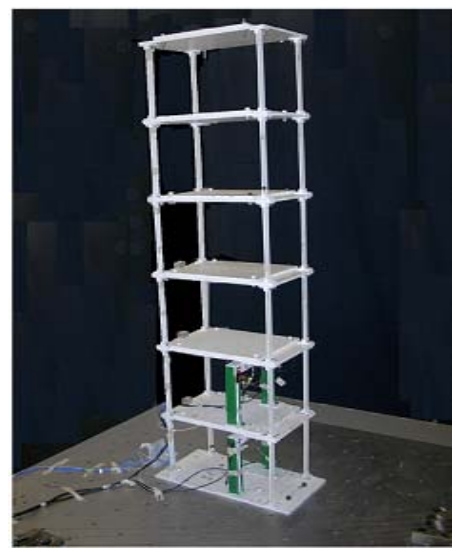

Fig. 1. Photograph of the test structure

\section{EXPERIMENTAL SETUP}

The theoretical and experimental studies are done on a semiactivelly controlled structure which is designed and constructed by the the Washington University Structural Control and Earthquake Engineering Laboratory (WUSCEEL), as shown in the figure 1. The test structure is of 6 stories, single bay, steel frame and $188 \mathrm{~cm}$ tall and has a mass of $147 \mathrm{~kg}$, distributed uniformly among the floors. A couple of MR dampers is installed between the ground and first floor, and the other couple between the first and second floors of the structure. Control forces applied to the structure are sensed by means of force transducers placed in series with the MR damper. Absolute accelerations are measured at each floor of the structure by means of capacitive accelerometers. Data acquisition, control actions and system evaluation are realized through a DSP-based real time control system fabricated by dSpace, Inc., which includes a 16-bit 16-analog input PC board (DSP2003) and a 16-bit 8-analog output PC board (DSP2101). Ground 
excitations are obtained by means of a uniaxial seismic simulator, which consists of a $1.5 \mathrm{~m} \times 1.5 \mathrm{~m}$ aluminium sliding table (PEGASUS) mounted on high-precision, low friction, linear bearings.

The MR damper used in the experiments is a prototype device fabricated by the Lord Corporation for testing and evaluation. It consists of two steel parallel plates (see figure 2) with the dimension of $4.45 \mathrm{~cm} \times 1.9 \mathrm{~cm} \times 2.5 \mathrm{~cm}$. Damper force is generated when the moving plate, coated with a thin foam saturated with MR fluid, slides between the two parallel plates. An electromagnet, consisting of a coil installed at one end of the devices, produces the magnetic field applied on the MR fluid of the saturated foam. The center plate of the device is $0.495 \mathrm{~cm}$ thick, resulting in a gap of $0.071 \mathrm{~cm}$. Thus, a maximum force of $29 \mathrm{~N}$ can be generated by each device, which is approximately $1.6 \%$ the weight of the structure. Electric power is supplied to the device by means of a current amplifier in which an output DC current between $0 \sim 1.2 A$ is present when an input voltage between $0 \sim 4 V$ is applied.

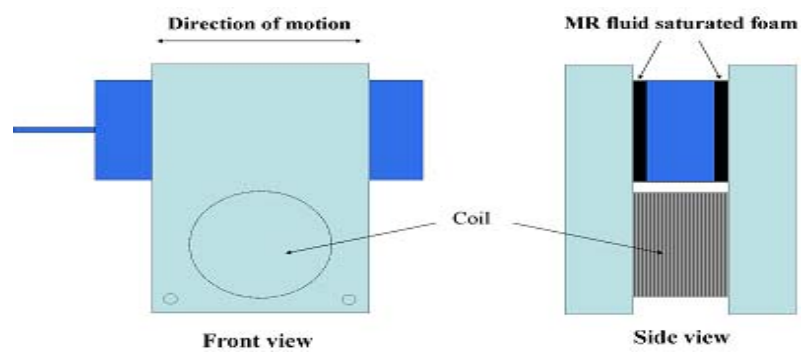

Fig. 2. Schematic diagram of shear mode MR damper

The dynamic motion of the structure is represented by the following equations:

$$
\boldsymbol{M}_{s} \ddot{\boldsymbol{x}}+\boldsymbol{C}_{s} \dot{\boldsymbol{x}}+\boldsymbol{K}_{s} \boldsymbol{x}=\boldsymbol{\Lambda} \boldsymbol{f}-\boldsymbol{M}_{s} \boldsymbol{\Gamma} \ddot{x}_{g}
$$

with $\boldsymbol{x}$ being a vector of relative displacements of the structural floors, $\ddot{x}_{g}$ the ground acceleration, $\boldsymbol{f}=$ $\left[f_{1}, f_{2}, \ldots, f_{m}\right]^{T}$ a vector of measured control forces generated by the $n t h$ MR dampers, $\Gamma$ a column vector of ones, and $\Lambda$ a vector determined by the placement of the MR dampers in the structure. Rewrite the equation (1) into the following state equation:

$$
\dot{z}=A z+B f+E \ddot{x}_{g} \quad ; \quad y=C z+D f
$$

where $\boldsymbol{z}=[\boldsymbol{x}, \dot{\boldsymbol{x}}]^{T}$ is a state vector and $\boldsymbol{y}=$ is vector of measured outputs about the absolute structural accelerations $\ddot{x}_{i}(i=1,2, \ldots, 6)$ and the semiactive control forces $f_{j}(j=$ $1,2,3,4)$ generated by the MR dampers, to which a voltage $u_{i} \in\left[0, u_{\max }\right]$ is applied through the current driver. From the practical point of view, the unknown seismic excitation $\ddot{x}_{g}(t)$ can be assumed to be bounded by $\left|\ddot{x}_{g}(t)\right| \leq X_{0}$ for all $t \geq 0$ where $X_{0}$ is a known positive constant according to the historical seismic records.

\section{SYSTEM IDENTIFICATION}

\section{A. Identification of test structure}

The available information of the test structure is the acceleration measurements of the ground and the ith structural floor. In order to identify the dynamics of the test structure, hybrid identification strategy is used, in which an analytical model is updated by using identified modal parameters and optimization algorithms. First, a white noise acceleration signal is used to excite the structure at the ground level. Then, the experimental transfer functions from the ground to the ith floor are obtained. The Eigensystem Realization Algorithm (ERA) [3] is applied to estimate the dynamic properties of the experimental structure (i.e. damping factors and natural frequencies). The finite impulse responses for each floor are required as inputs for the algorithm. Such responses are computed by applying the Inverse Fast Fourier Transform (IFFT) on each transfer function. The mass and stiffness parameters of the analytical model are optimized by using the identified damping factors and natural frequencies. The optimal damping matrix is found by using the FMINCON optimization function of MATLAB with objective function being defined as the sum of the square errors between the experimental and simulated acceleration values at each time sample, when a white noise excitation signal is applied to the ground level during $n$ seconds. The damping matrix is computed based on the method proposed in [3]:

$$
\boldsymbol{C}_{s}=\boldsymbol{M}_{s} \boldsymbol{\Phi} \operatorname{diag}\left(2 \boldsymbol{h}_{e}\left[2 \pi \boldsymbol{f}_{e}\right]\right) \boldsymbol{\Phi}^{T}
$$

with $\boldsymbol{\Phi}=\left[\begin{array}{llll}\phi_{1} & \phi_{2} & \ldots & \phi_{n}\end{array}\right]$ being the modal matrix, $\phi_{i}$ the eigenvectors of $\boldsymbol{M}_{s}^{-1} \boldsymbol{K}_{s}, \boldsymbol{f}_{e}$ are the frequencies and $\boldsymbol{h}_{e}$ the damping factors estimated by using the ERA. The initial parameters used to optimize the damping matrix correspond to the damping factors, while natural frequencies are maintained constant during the optimization.

By using the above procedure, the following estimated values of the test structure are obtained: stiffness of each floor $k_{i}=273 \mathrm{~N} / \mathrm{cm}$ and mass $m_{i}=0.227 \mathrm{Ns}^{2} / \mathrm{cm}$. The natural damping factor has been assumed to be $1 \%$ for each floor and the natural frequencies obtained by means of the analytical model are: [1.39 4.086 .548 .6210 .1911 .18$] \mathrm{Hz}$, while the natural frequencies and damping factors estimated by using ERA are: [1.29 3.856 .118 .229 .6410 .81$] \mathrm{Hz}$ and [ $\left.\begin{array}{llllll}1.38 & 0.71 & 0.64 & 0.56 & 0.48 & 0.91\end{array}\right]$ (\%). Figure 3 presents a plot of the transfer function (from ground acceleration to the fourth floor) obtained by using the experimental data, the analytical model, and the model with optimized damping and mass parameters. Finally, the optimal damping factors $\left[\begin{array}{llllll}4.95 & 1.16 & 0.76 & 0.41 & 0.20 & 0.24\end{array} \%\right.$ are obtained by using the white noise as excitation signal during 60 seconds. 


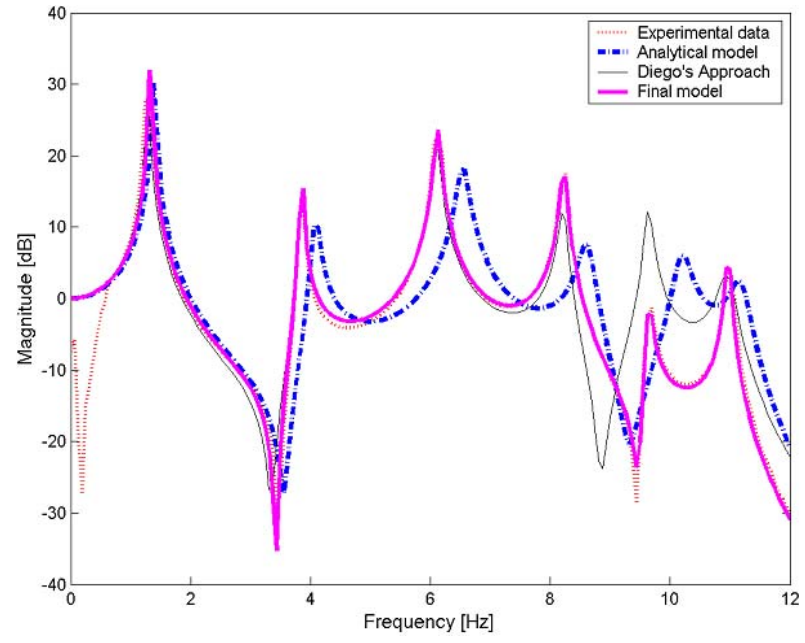

Fig. 3. Analytical and experimental transfer functions from ground to fourth floor acceleration

\section{B. Identification of MR dampers}

A simple mechanical model for the MR damper has been previously developed in [4], [5] in which experimental tests show that it can predict accurately the behavior of a MR damper with the advantage of being adequate for control purposes. The MR damper force is expressed as follows:

$$
\begin{aligned}
f & =\delta \Delta \dot{q}+\alpha z \\
\dot{z} & =-\gamma|\Delta \dot{q}| z|z|^{n-1}-\beta \Delta \dot{q}|z|^{n}+A_{a} \Delta \dot{q}
\end{aligned}
$$

with $\Delta q=q_{i}-q_{j}$ being the difference of displacement at the ends of the device and $z$ an evolutionary variable that accounts for the dependence of the historical response. The parameters $\gamma, \beta, n$ and $A_{a}$ can be adjusted to control the linearity in the unloading situation and the smoothness of the transition from the pre-yield to the post-yield region [6], [7], [8]. The parameters of a MR damper depend generally on the commanding voltage signal $u$. Thus, for control purposes this dependence is formulated as

$$
\alpha=\alpha(u)=\alpha_{a}+\alpha_{b} u \quad \delta=\delta(u)=\delta_{a}+\delta_{b} u
$$

The dynamic response of the current driver circuit to changes in the command input is approximated by a firstorder time lag expressed by:

$$
\dot{u}=-\eta(u-v)
$$

where $v$ is the command voltage applied to the control circuit.

The identification of MR dampers can be done in two steps. First, the MR damper parameters are identified before being installed at the structure. For this purpose, an experimental frame is used in which the measurements of the structural displacement and velocity and the damper force are used to identify the parameters of the model expressed in equations (4)-(7). Afterwards, when MR dampers are installed at the structure, their parameters will be updated. In this case, a variety of representative tests are realized to update the MR damper parameters by using sinusoidal excitations with different frequencies and amplitudes being applied at the ground level of the structure. Concretely, three configurations are studied: (1) Two MR dampers installed between the base and the first floor (2) Two MR dampers installed between the first and second floor and (3) two MR dampers on each of the fist two floors of the structure. Forces generated by each MR damper and accelerations induced to each floor are measured in order to identify and optimize the MR damper parameters. The FMINCON optimization function is used to determine the optimal values by taking the values obtained in the step 1 as the initial values. The objective function is defined as the error between the experimental and predicted accelerations at each floor. Predicted responses are calculated by using the optimal $M_{s}, C_{s}$ and $K_{s}$ matrices. As a result, the following optimal MR damper parameters are obtained: $\delta_{a}=0.0454$; $\delta_{b}=0.0195 ; A_{a}=12 ; \gamma=300 ; \beta=300 ; \eta=80$; while the values of $\alpha_{a}$ and $\alpha_{b}$ of the four MR dampers are varied in the ranges of $\alpha_{a} \in[45,60]$ and $\alpha_{b} \in[45,90]$. Finally, an integrated system model is obtained by using the optimal parameters of the structure and the MR damper after their installation. In figure 4, it is shown the good result of identification obtained by comparing the experimental and analytical MR damper forces.

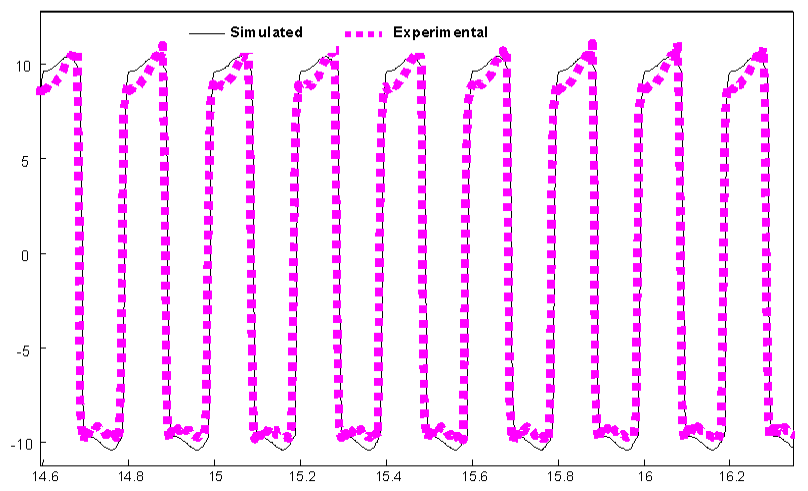

Fig. 4. Experimental and simulated MR damper forces

\section{CONTROLLER DESIGN}

The control objective is to design a semiactive controller that can effectively attenuate structural vibrations when uncertain disturbances act on the structure. In this paper, the backstepping control technique is used for the design of semiactive controller, in which the hysteretic dynamics of the MR dampers is taken into account. Based on the motion equations of the test structure for the nodes 1 and 2 where the MR dampers are installed,

$$
\begin{aligned}
& \ddot{x}_{1}=-\frac{1}{m_{1}}\left[\sum_{i=2}^{n} m_{i} \ddot{x}_{i}+\sum_{i=1}^{n} m_{i} \ddot{x}_{g}+k_{1} x_{1}+c_{1} \dot{x}_{1}+f_{1}\right] \\
& \ddot{x}_{2}=-\frac{1}{m_{2}}\left[\sum_{i=3}^{n} m_{i} \ddot{x}_{i}-k_{1} x_{1}-c_{1} \dot{x}_{1}+\left(k_{1}+k_{2}\right) x_{2}+\right.
\end{aligned}
$$




$$
\left.\left(c_{1}+c_{2}\right) \dot{x}_{2}+f_{2}+\sum_{i=2}^{n} m_{i} \ddot{x}_{g}\right]
$$

the following state equations are obtained:

$$
\begin{aligned}
\dot{y}_{1} & =y_{2} \\
\dot{y}_{2} & =-\frac{1}{m_{1}}\left[\sum_{i=2}^{n} m_{i} \ddot{x}_{i}+\sum_{i=1}^{n} m_{i} \ddot{x}_{g}+k_{1} y_{1}+c_{1} y_{2}+f_{1}\right] \\
\dot{y}_{4} & =-\frac{1}{m_{2}}\left[\sum_{i=3}^{n} m_{i} \ddot{x}_{i}-k_{1} y_{1}-c_{1} y_{2}+\left(k_{1}+k_{2}\right) y_{3}\right. \\
& \left.+\left(c_{1}+c_{2}\right) y_{4}+f_{2}+\sum_{i=2}^{n} m_{i} \ddot{x}_{g}\right]
\end{aligned}
$$

(8) (12) and (13) with the estimated value of the evolutionary $z$. The second one uses the modified Clipped-Optimal control algorithm, used in [4], to compute the equivalent command voltage.

1) Backstepping control scheme 1 (BE $\left.E_{1}\right)$ : By using (4) and (6) and the desired force values $f_{1}$ and $f_{2}$ obtained in (12) and (13), the following control law is obtained:

$$
u_{i}=\frac{f_{i}-\alpha_{a_{i}} z_{i}+\delta_{a_{i}} \Delta y_{i}}{\alpha_{b_{i}} z_{i}+\delta_{b_{i}} \Delta y_{i}} ; \quad i=1,2
$$

(9) where $\Delta y_{1}=y_{2}$ and $\Delta y_{2}=y_{4}-y_{2}$. Since the variable $z_{i}$ cannot be measured directly, an estimated value $\hat{z}_{i}$ is obtained:

$$
\dot{\hat{z}}_{i}=-\gamma_{i}\left|\Delta y_{i}\right| \hat{z}_{i}\left|\hat{z}_{i}\right|^{n-1}-\beta_{i} \Delta y_{i}\left|\hat{z}_{i}\right|^{n}+A_{i} \Delta y_{i}
$$

The following standard variables, typically adopted in the literature of backstepping control [9]-[10], are used for the controller design:

$$
\begin{array}{lll}
e_{1}=y_{1} ; & \dot{e}_{1}=y_{2} ; & e_{1} \dot{e}_{1}=e_{1} y_{2} ; \\
e_{2}=y_{2}-\alpha_{1} ; & \dot{e}_{2}=\dot{y}_{2}+h_{1} y_{2} ; & e_{2} \dot{e}_{2}=e_{2}\left(\dot{y}_{2}+h_{1} y_{2}\right) \\
e_{3}=y_{3} ; & \dot{e}_{3}=y_{4} ; & e_{3} \dot{e}_{3}=e_{3} y_{4} ; \\
e_{4}=y_{4}-\alpha_{2} ; & \dot{e}_{4}=\dot{y}_{4}+h_{3} y_{4} ; & e_{4} \dot{e}_{4}=e_{4}\left(\dot{y}_{4}+h_{3} y_{4}\right) \\
\alpha_{1}=-h_{1} e_{1} & \alpha_{2}=-h_{3} e_{3} ; &
\end{array}
$$

By substituting (9) into the last equation, one obtains:

$$
\begin{aligned}
e_{2} \dot{e}_{2}= & -\frac{e_{2}}{m_{1}}\left[\sum_{i=2}^{n} m_{i} \ddot{x}_{i}+k_{1} y_{1}+\left(c_{1}-m_{1} h_{1}\right) y_{2}+f_{1}\right. \\
& \left.+\sum_{i=1}^{n} m_{i} \ddot{x}_{g}\right] \\
e_{4} \dot{e}_{4}= & -\frac{e_{4}}{m_{2}}\left[\sum_{i=3}^{n} m_{i} \ddot{x}_{i}-k_{1} y_{1}-c_{1} y_{2}+\left(k_{1}+k_{2}\right) y_{3}\right. \\
& \left.+\left(c_{1}+c_{2}-m_{2} h_{3}\right) y_{4}+\sum_{i=2}^{n} m_{i} \ddot{x}_{g}+f_{2}\right]
\end{aligned}
$$

In order to achieve the asymptotic error suppression, the following control law is derived

$$
\begin{aligned}
f_{1}= & -\sum_{i=2}^{n} m_{i} \ddot{x}_{i}-\sum_{i=1}^{n} m_{i} \ddot{x}_{g}-k_{1} y_{1}-\left(c_{1}-m_{1} h_{1}\right) y_{2} \\
& +m_{1} e_{1} \\
f_{2}= & -\sum_{i=3}^{n} m_{i} \ddot{x}_{i}-\sum_{i=2}^{n} m_{i} \ddot{x}_{g}+k_{1} y_{1}+c_{1} y_{2}-\left(k_{1}+\right. \\
& \left.k_{2}\right) y_{3}-\left(c_{1}+c_{2}\right) y_{4}+m_{2} e_{3}
\end{aligned}
$$

However, the control laws (12) and (13) are not implementable in practice since they contain unmeasurable variables, such as $z$ and $\ddot{x}_{g}$. On the other hand, a voltage command, in stead of a force command, is required for the MR dampers. In order to overcome these problems, two semiactive backstepping control approaches are studied. The first one computes the equivalent command voltage based on the equation (4), the force value obtained from
Define $\tilde{z}_{i}=z_{i}-\hat{z}_{i}$ as the estimation error between the real value $z_{i}$ and the estimated value $\hat{z}_{i}$, then

$$
z_{i}=\tilde{z}_{i}+\hat{z}_{i} ; \quad \dot{\tilde{z}}_{i}=\dot{z}_{i}-\dot{\hat{z}}_{i}
$$

By taking $\tilde{z}_{1}=e_{2}, \tilde{z}_{2}=e_{4}$, the denominator of the commanding voltage signals $u_{1}$ and $u_{2}$ can be replaced by $\delta_{b_{1}} y_{2}+\alpha_{b_{1}} \hat{z}_{1}+\alpha_{b_{1}} \tilde{z}_{1}=\delta_{b_{1}} y_{2}+\alpha_{b_{1}} \hat{z}_{1}+\alpha_{b_{1}} e_{2}$ and $\delta_{b_{2}}\left(y_{4}-y_{2}\right)+\alpha_{b_{2}} \hat{z}_{2}+\alpha_{b_{2}} \tilde{z}_{2}=\delta_{b_{2}}\left(y_{4}-y_{2}\right)+\alpha_{b_{2}} \hat{z}_{2}+\alpha_{b_{2}} e_{4}$. Now, an implementable law, based upon the bounded values of $X_{0}$ and the estimated values of $z_{i}$, is adopted for the backstepping control:

$$
\begin{aligned}
u_{1}= & \frac{1}{\alpha_{b_{1}}\left(\hat{z}_{1}+e_{2}\right)+\delta_{b_{1}} y_{2}}\left[-\sum_{i=2}^{n} m_{i} \ddot{x}_{i}-k_{1} y_{1}-\alpha_{a_{1}} z_{1}\right. \\
& \left.-\left(\delta_{a_{1}}-m_{1} h_{1} c_{1}\right) y_{2}+m_{1} e_{1}-\sum_{i=1}^{n} m_{i} X_{0} \operatorname{sgn}\left(e_{2}\right)\right] \\
u_{2}= & \frac{1}{\alpha_{b_{2}}\left(\hat{z}_{2}+e_{4}\right)+\delta_{b_{2}}\left(y_{4}-y_{2}\right)}\left[-\sum_{i=3}^{n} m_{i} \ddot{x}_{i}+k_{1} y_{1}\right. \\
& +\left(c_{1}+\delta_{a_{2}}\right) y_{2}-\left(k_{1}+k_{2}\right) y_{3}-\left(c_{1}+c_{2}+\delta_{a_{2}}\right) y_{4} \\
& \left.-\alpha_{a_{2}} z_{2}+m_{2} e_{3}-\sum_{i=2}^{n} m_{i} X_{0} \operatorname{sgn}\left(e_{4}\right)\right]
\end{aligned}
$$

for all $\alpha_{b_{1}}\left(\hat{z}_{1}+e_{2}\right)+\delta_{b_{1}} y_{2} \neq 0$ and $\alpha_{b_{2}}\left(\hat{z}_{2}+e_{4}\right)+$ $\delta_{b_{2}}\left(y_{4}-y_{2}\right) \neq 0$, otherwise $u_{i}=0$. Moreover, for some types of MR dampers [6]-[8], the constraints $\gamma \geq \beta \geq 0$ and $n=1$ must be satisfied by the control law.

\section{Stability Analysis}

In order to verify the closed-loop stability, the following Lyapunov function candidate is defined:

$$
\begin{aligned}
& V=\frac{1}{2} e_{1}^{2}+\frac{1}{2} e_{2}^{2}+\frac{1}{2} e_{3}^{2}+\frac{1}{2} e_{4}^{2}+\frac{1}{2} \tilde{z}_{1}^{2}+\frac{1}{2} \tilde{z}_{2}^{2} \\
& \dot{V}=e_{1} \dot{e}_{1}+e_{2} \dot{e}_{2}+e_{3} \dot{e}_{3}+e_{4} \dot{e}_{4}+\tilde{z}_{1} \dot{\tilde{z}}_{1}+\tilde{z}_{2} \dot{\tilde{z}}_{2}
\end{aligned}
$$


From equations (11)-(13), (17)-(17), one obtains:

$$
\begin{aligned}
e_{1} \dot{e}_{1} & =e_{1} y_{2} \quad e_{3} \dot{e}_{3}=e_{3} y_{4} \\
e_{2} \dot{e}_{2} & =-\frac{1}{m_{1}} \sum_{i=1}^{n} m_{i}\left[X_{0}\left|e_{2}\right|-\ddot{x}_{g} e_{2}\right]-e_{1} e_{2}-h_{2} e_{2}^{2} \\
e_{4} \dot{e}_{4} & =-\frac{1}{m_{2}} \sum_{i=2}^{n} m_{i}\left[X_{0}\left|e_{4}\right|-\ddot{x}_{g} e_{4}\right]-e_{3} e_{4}-h_{4} e_{4}^{2}
\end{aligned}
$$

with $h_{2}=m_{1}^{-1} \alpha_{a_{1}}$ and $h_{4}=m_{2}^{-1} \alpha_{a_{2}}$.

From equations (5), (15)-(16) and for $\mathrm{n}=1$ one gets:

$$
\begin{aligned}
\tilde{z}_{i} \dot{\tilde{z}}_{i} & =-\gamma_{i}\left|\Delta y_{i}\right| \tilde{z}_{i}^{2}-\beta_{i} \Delta y_{i} \tilde{z}_{i}\left(\left|z_{i}\right|-\left|\hat{z}_{i}\right|\right) \\
& \leq-\left(\gamma_{i}-\beta_{i}\right)\left|\Delta y_{i}\right| \tilde{z}_{i}^{2} \leq 0
\end{aligned}
$$

Finally, the derivative of Lyapunov function becomes:

$$
\begin{aligned}
\dot{V}= & -\frac{1}{m_{1}} \sum_{i=1}^{n} m_{i}\left[X_{0}\left|e_{2}\right|-\ddot{x}_{g} e_{2}\right]-h_{2} e_{2}^{2}-h_{4} e_{4}^{2} \\
& -\frac{1}{m_{2}} \sum_{i=2}^{n} m_{i}\left[X_{0}\left|e_{4}\right|-\ddot{x}_{g} e_{4}\right]-\left(\gamma_{1}-\beta_{1}\right)\left|y_{2}\right| \tilde{z}_{1}^{2}- \\
& \left(\gamma_{2}-\beta_{2}\right)\left|y_{4}-y_{2}\right| \tilde{z}_{2}^{2} \leq 0
\end{aligned}
$$

Therefore, the stability of the closed-loop system is ensured.

2) Backstepping control scheme $2\left(B E_{2}\right)$ : The control approach consists of using the desired forces $f_{1}$ and $f_{2}$ obtained in (12) and (13) to obtain an equivalent command voltage through the modified clipped-optimal technique. This technique was proposed for controlling a single MR damper [6] and multiple MR devices [7], and was experimentally verified in [5]. This control approach is graphically represented in figure 5. The algorithm consists in appending $m$ force feedback loops to induce each MR damper and to produce approximately a desired control force. Then, a command voltage signal is obtained as follows: when the ith MR damper provides the desired optimal force (i.e. $f_{i}=f_{c_{i}}$ ) the voltage applied to the MR damper should remain at the present value. If the magnitude of the desired optimal force is between the minimal force $f_{0_{i}}$ and the maximum force $f_{\text {max }_{i}}$, and the two forces have the same sign, the voltage applied to the current driver is derived from a linear relation, experimentally obtained, between the output force and the input voltage which takes the form: $f_{i}=f_{0_{i}}+m\left(u_{i}-u_{0_{i}}\right)$. Otherwise, the commanded voltage is set to zero. Thus, the control law for the ith MR damper, using the modified clipped-optimal control, is given as follows:

$\begin{cases}u_{i}=u_{0_{i}}+m^{-1}\left(f_{i}-f_{0_{i}}\right) & \text { if }\left\{\begin{array}{l}\operatorname{sgn}\left(f_{i}\right)=\operatorname{sgn}\left(f_{c_{i}}\right) \\ \left|f_{0_{i}}\right| \leq\left|f_{c_{i}}\right| \leq\left|f_{i_{\text {max }}}\right| \\ u_{i}=u_{\max }\end{array}\right. \\ \begin{array}{ll}\operatorname{sgn}\left(f_{i}\right)=\operatorname{sgn}\left(f_{c_{i}}\right) \\ \left|f_{c_{i}}\right|>\left|f_{i_{\text {max }}}\right|\end{array} \\ u_{i}=0 & \text { otherwise }\end{cases}$

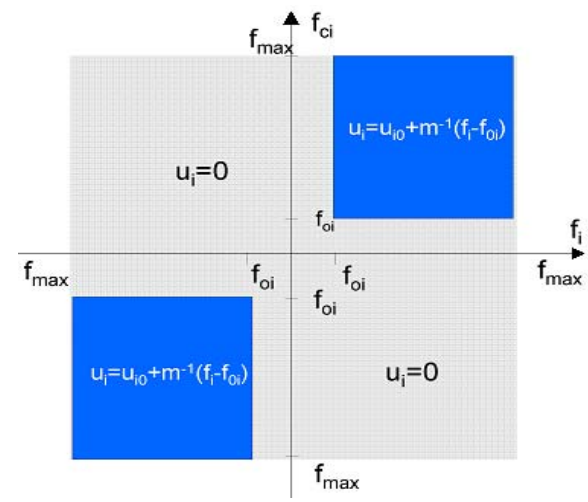

Fig. 5. Graphical representation of the Clipped-Optimal control technique

\section{EXPERIMENTAL RESULTS}

The experiments of semiactive vibrational control were implemented on a 6-story test structure installed in the WUSCEEL. The El Centro earthquake has been used as the excitation signal for verifying the effectiveness of proposed backstepping controllers and for comparing the semiactive control case with the passive-on case (with the maximal damping coefficient). In Figure 6, the peak acceleration response of the third floor is given to show the effectiveness of the proposed backstepping control scheme.

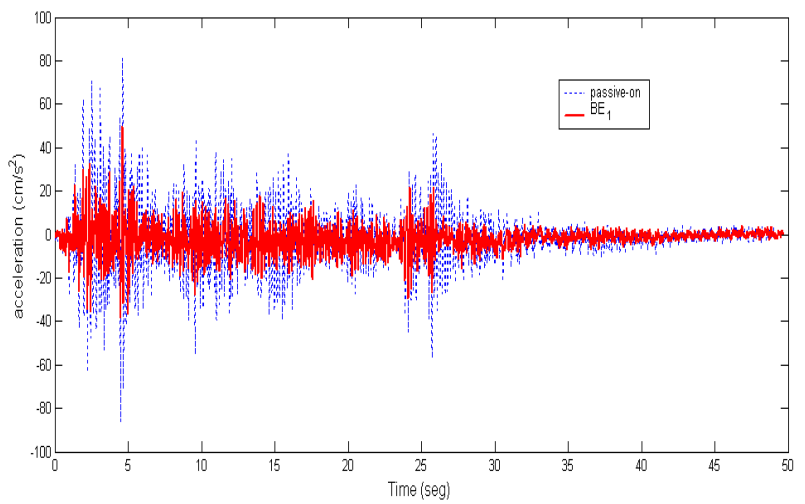

Fig. 6. Third floor acceleration response in presence of El Centro earthquake

Quantitative evaluation of the control performance has been made by using five evaluation criteria [11], related to the normalized and peak floor accelerations and base shear, and the maximum control force between the two MR dampers, respectively.

The first evaluation criterion considers the normalized peak response of absolute floor accelerations

$$
J_{1}=\max _{i, t}\left(\frac{\left|\ddot{x}_{a i}(t)\right|}{\ddot{x}_{a}^{\text {max }}}\right)
$$

where the absolute acceleration of the ith floor of the structure $\ddot{x}_{a i}(t)$ is normalized by the peak uncontrolled floor acceleration, denoted by $\ddot{x}_{a}^{\max }$. 
The second evaluation criterion considers the normalized peak acceleration response

$$
J_{2}=\max _{i, t}\left(\frac{\left\|\ddot{x}_{a i}(t)\right\|}{\left\|\ddot{x}_{a}^{\max }\right\|}\right)
$$

where $\left\|\ddot{x}_{a i}(t)\right\|=\sqrt{\int_{0}^{t f} \ddot{x}_{a i}^{2}(t) d t}$ and the absolute accelerations of the $i t h$ floor $\ddot{x}_{a i}(t)$ are normalized by the peak uncontrolled floor acceleration, denoted by $\left\|\ddot{x}_{a}^{\max }\right\|$.

The third evaluation criterion considers the maximum base shear generated in the controlled configuration

$$
J_{3}=\max _{t}\left|\sum_{i=1}^{6} \frac{m_{i} \ddot{x}_{a i}(t)}{F_{b}^{\text {max }}}\right|
$$

where $F_{b}^{\max }$ describes the maximum base shear in the uncontrolled configuration.

The fourth evaluation criterion corresponds to the normed/nondimensionalized base shear.

$$
J_{4}=\frac{\left\|\sum_{i=1}^{6} m_{i} \ddot{x}_{a i}(t)\right\|}{\left\|F_{b}^{\text {max }}\right\|}
$$

where $\left\|F_{b}^{\text {max }}\right\|=\left\|\sum_{i=1}^{6} m_{i} \ddot{x}_{a i}(t)\right\|$ represents the maximum normalized uncontrolled base shear.

Finally, the fifth evaluation criterion is a measure of the maximum control force per device,

$$
J_{5}=\max _{t, i}\left(\frac{\left|f_{i}(t)\right|}{W}\right)
$$

where $f_{i}(t)$ is the force generated by the $i t h$ control device over the time story of each earthquake and $\mathrm{W}=1446 \mathrm{~N}=$ weight of the structure.

In the following table, the normalized experimental responses are shown for the passive-on and semiactive control cases.

TABLE I

NORMALIZED EXPERIMENTAL RESPONSES

\begin{tabular}{lccccc}
\hline \hline Control strategy & $J_{1}$ & $J_{2}$ & $J_{3}$ & $J_{4}$ & $J_{5}$ \\
\hline \hline Passive-on & 0,8159 & 0,4256 & 1,1107 & 0,5711 & 0,0499 \\
$\mathrm{BE}_{2}$ & 0,6252 & 0,4080 & 0,8519 & 0,7654 & 0,0432 \\
$\mathrm{BE}_{1}$ & 0,6554 & 0,3432 & 0,8063 & 0,4298 & 0,0078 \\
\hline \hline
\end{tabular}

It is seen from the Table 1 that the dynamic performance of the structure has been enhanced by using backstepping controllers as compared with the passive-on case.

\section{CONCLUSIONS}

In this paper, two new semiactive control schemes have been proposed and experimentally verified for the vibration attenuation of civil engineering structures, in the first one by estimating the unmeasurable and in the second one by using the modified clipped-optimal algorithm. In the controller design, the hysteretic dynamics of semiactive MR dampers have been taken into account. In this way, the proposed controllers have presented a better performance in real operation conditions. The experimental verification for a 6-story structure has shown the effectiveness of the semiactive backstepping controllers proposed.

\section{REFERENCES}

[1] N. Luo, J. Rodellar and M. De la Sen, Composite robust active control of seismically excited structures with actuator dynamics, Earthquake Engineering and Structural Dynamics, 27,1998, pp. 301311.

[2] N. Luo, J. Rodellar, J. Vehí and M. De la Sen, Composite Semiactive control of a class of seismically excited structures, Special Issue on Control of Structural and Mechanical System, Journal of The Franklin Institute, 338, 3, 2001, pp. 225-240.

[3] D. Giraldo, O. Yoshida, S.J. Dyke and L. Giacosa, Control oriented system identification using ERA, Journal of Structural Control and Health Monitoring, 2004.

[4] Yoshida, O., Dyke, S. J., Giacosa, L. M. and Truman, K. Z., Experimental verification of torsional response control of asymmetrics buildings, Earthquake Engineering and Structural Dynamics, 32, 2003, pp. 2085-2105.

[5] F. Yi, S.J. Dyke ,J.M. Caicedo and J.D. Carlson, Experimental verification of multi-input seismic control strategies for smart dampers, Journal of Engineering Mechanics, ASCE, 2004, pp. 1152-1164.

[6] S.J. Dyke, B.F. Spencer Jr., M.K. Sain and J.D. Carlson, Modeling and control of magnetorheological dampers for seismic response reduction, Smart Materials and Structures, Vol. 5, 1996, pp. 565575.

[7] S.J. Dyke and B.F. Spencer Jr., "Seismic response control using multiple MR dampers", Proceedings of the 2nd International Workshop on Structural Control, Hong Kong, December, 1996, pp.163-173.

[8] B.F. Spencer Jr., S.J. Dyke and J.D. Carlson Phenomenological model for magnetorheological dampers, Journal of Engineering Mechanics, ASCE, 123, No. 3, pp. 230-238. 1997.

[9] F. Ikhouane and M. Krstic, Adaptive backstepping with parameter projection: robustness and asymptotic performance, Automatica, 34, 1998, pp. 49-435.

[10] F. Ikhouane, V. Manosa and J. Rodellar, Adaptive backstepping control of a class of uncertain nonlinear systems. Application to BoucWen hysteretic oscillators, Preprint Centre de Recerca Matemàtica, $\mathrm{N}-535,2003$.

[11] B.F. Spencer Jr., S.J. Dyke and H.S. Deoskar, "Benchmark problems in structural control, Part I: Active Mass Driver System", Proceedings of the ASCE Structural Congress, Oregon, 1997. 